\title{
Estudo da atividade estelar da Kepler-289 a partir da modelagem de trânsitos planetários
}

\author{
Study of stellar activity of Kepler-289 from the modeling of planetary transits \\ Alexandre Araujo de Souza*1], Adriana Valio ${ }^{10}$ \\ ${ }^{1}$ Universidade Presbiteriana Mackenzie, São Paulo, SP, Brasil
}

Recebido em 27 de Novembro, 2018. Revisado em 11 de Abril, 2019. Aceito em 13 de Maio, 2019.

\begin{abstract}
Neste trabalho apresentamos um estudo sobre atividade estelar e trânsitos planetários de exoplanetas. Sem apresentar rigor matemático demostramos como obter parâmetros físicos como: temperatura, intensidade e posição de manchas em superfície de estrelas do tipo solar, bem como, a rotação estelar média a partir de dados públicos da Missão Espacial Kepler. Como exemplo, usamos as curvas de luz da estrela Kepler-289 e modelamos o trânsito do planeta Kepler-289d, resultando em 9 manchas detectadas e uma rotação estelar média de 8,76 dias.
\end{abstract}

Palavras-chave: manchas estelares, exoplanetas, rotação.

\begin{abstract}
We present a study of stellar activity and planetary transits of exoplanets. Without showing a mathematical rigor we showed how to obtain physical parameters such as: temperature, intensity and position of spots on the surface of solar type stars, as well as the average stellar rotation from public data of the Kepler Space Mission. As an example, we used the light curves of the Kepler-289 star and modeled the transits of the planet Kepler-289d resulting in 9 detected spots and a mean stellar rotation of 8.76 days.
\end{abstract}

Keywords: starspot, exoplanet, rotation.

\section{Introdução}

A partir de novas instrumentações astronômicas e lançamentos de missões espaciais, a Astronomia tem reportado com frequência novas descobertas acerca de diversos fenômenos existentes no universo. Tais descobertas proporcionam o surgimento de técnicas cada vez mais precisas e eficazes no estudo de diversas áreas do conhecimento astronômico. A Astrofísica Estelar, área da Astronomia que se dedica ao estudo das estrelas, tem relatado grandes descobertas com o surgimento de missões espaciais como: a CoRoT (COnvection ROtation et Transits planétaires), Kepler, TESS (Transiting Exoplanet Survey Satellite) e a futura missão PLATO (PLAnetary Transits and Oscillations of stars). Os resultados obtidos com essas missões são de grande importância para o entendimento sobre a formação do nosso sistema solar, a relação da estrela com seus planetas e sobre a formação dos diversos planetas existentes. Neste trabalho, o destaque será para a missão espacial Kepler da NASA que obteve grande êxito na descoberta de planetas fora do sistema solar.

A missão Kepler lançada em 2009 foi a $10^{\mathrm{a}}$ missão da NASA na classe "Descoberta". O principal objetivo científico da missão Kepler era a detecção de exoplanetas pelo método de trânsitos planetários, com ênfase em planetas terrestres (Raio <2.5 Raio da Terra) localizados

*Endereço de correspondência: adesouza.astro@gmail.com dentro das zonas habitávei 1 das estrelas semelhantes ao Sol [1]. Atingir essa meta científica exigiu observar um único grande campo de visão de 115 graus em direção à constelação de Cisne. A missão obteve grandes descobertas na área de exoplanetas (por exemplo, [2]), e foi igualmente produtiva ao estudar atividade estelar. A Astrossismologia com Kepler forneceu informações sobre a estrutura interna das estrelas além do nosso Sol, que coloca restrições em suas massas, raios e idades [3].

As curvas de luz do Kepler também permitiram que a rotação estelar fosse caracterizada para dezenas de milhares de estrelas $([4] ;[5])$. Estudos sobre explosões estelares também tiveram grandes destaques com dados da missão Kepler, como o estudo de flare $2^{2}$ e superflares em estrelas do tipo solar [6].

O telescópio Kepler (Figura 1) foi lançado em uma órbita heliocêntrica de 372,5 dias, da Estação da Força Aérea de Cabo Canaveral - EUA, a bordo de um Delta II 7925-10L em 2009, no dia 7 de março. Nesta órbita, Kepler observou quase continuamente um campo de visão que foi cuidadosamente selecionado para fornecer uma densidade apropriada de estrelas-alvo. No entanto, o

\footnotetext{
${ }^{1}$ Uma zona habitável é uma região em que a distância do planeta em relação à estrela possibilite a existência de temperatura adequada para que o planeta tenha água em forma líquida na sua superfície, para permitir o movimento das partículas e a eventual formação de moléculas orgânicas complexas, e fontes de energia (luz estelar, calor interno ou energia química) para manter o metabolismo.

${ }^{2}$ Explosões oriundas de regiões ativas na superfície das estrelas
} 


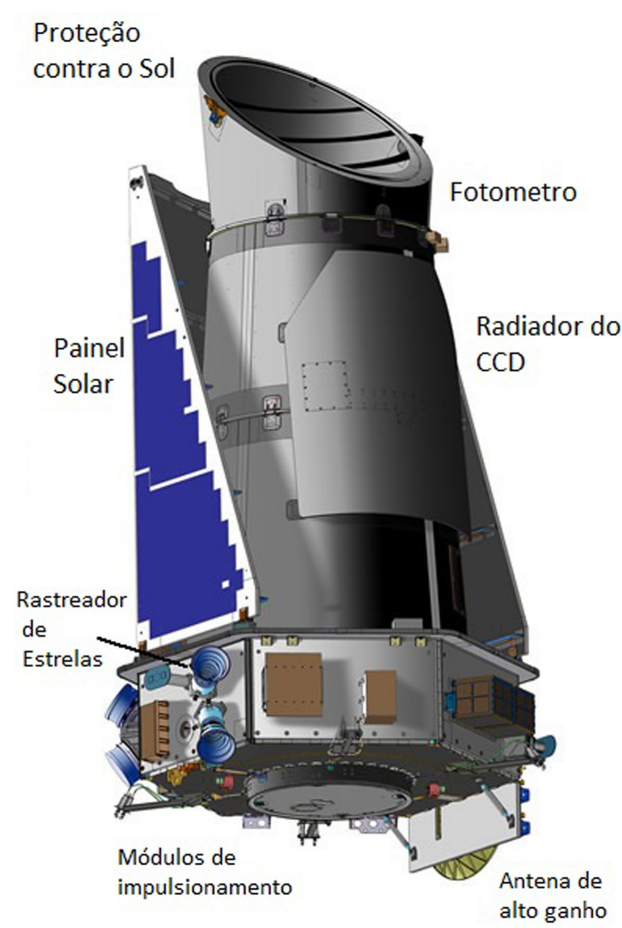

Figura 1: Representação artística do satélite Kepler. Crédito: NASA

Fotômetrd ${ }^{3}$ deve ser girado a $90^{\circ}$ em torno de seu eixo a cada 93 dias para manter os painéis solares iluminados e o radiador que resfria os painéis do plano focal apontados para longe do Sol [7].

O telescópio Kepler de uma tonelada tem um espelho primário de 1,44 metros, um fotômetro de 0,95 metros de diâmetro e um conjunto de sensores $\mathrm{CCD}^{4}$ com até 94.6 megapixels. O resultado das observações do telescópio Kepler é dado em forma de curva de luz. Uma curva de luz é definida como a evolução do brilho da estrela no decorrer do tempo, assim é possível visualizar qualquer variabilidade fotométrica da estrela. Dessa forma, por meio da análise e interpretação das curvas de luz, fenômenos físicos presentes nas estrelas podem ser identificados. As observações das estrelas-alvo da missão Kepler estão registradas ao longo de até 17 quarter $\$ 5$ (um quarto em português). Cada quarter tinha duração de 1 trimestre distribuídos em curvas de longa (1 ponto a cada 29,4 minutos) e curta cadência ( 1 ponto a cada 1 minuto). Estes dois tipos de curva de luz podem ser extraídos dos arquivos disponíveis no MAST ${ }^{6}$ (Mikulski Archive for Space Telescopes, em inglês).

Os dados do telescópio Kepler provaram ser um ótimo recurso para pesquisa de Atividade Estelar e fornecem um

\footnotetext{
3 Aparelho que mede a intensidade da luz

4 CCD (Charge Coupled Device) acopladas aos telescópios, que têm a função de converter fótons em elétrons.

${ }^{5} \mathrm{Um}$ quarter é o intervalo de 93 dias que o telescópio levava para rotacionar $90^{\circ}$ em torno de seu eixo para manter os painéis solares na direção do Sol.

6 https://archive.stsci.edu/index.html
}

conjunto de dados contínuos de precisão sem precedentes ao longo de quatro anos para um conjunto de quase 160.000 estrelas. Assim, a missão produziu uma grande diversidade na descoberta de exoplanetas, como: júpiteres quentes, netunos quentes, júpiteres frios, super júpiteres, super terras, exoterras. Além da grande contribuição para descobertas de novos planetas, a missão possibilitou o estudo da atividade estelar de estrelas do tipo solar, como também a possibilidade de estudar ciclos magnéticos, rotação diferencial e manchas na superfície destas estrelas [8].

O interesse desse artigo é apresentar ao leitor um estudo sobre a presença e identificação de características físicas de manchas na superfície de estrelas do tipo solar, e identificar a rotação estelar média de estrelas observadas pelo telescópio Kepler.

\section{O método de trânsito planetário}

O método de trânsitos planetários (Figura 2) é uma das técnicas utilizadas na descoberta de exoplanetas. O método de trânsitos planetários só funciona para sistemas estelares com planetas que têm órbitas alinhadas, de tal forma que, visto da Terra, o planeta cruza na frente do disco da estrela e bloqueia temporariamente uma pequena fração da luz vinda da mesma estrela. Atualmente o Método de Trânsitos Planetários é o mais eficaz na descoberta de exoplanetas, com 2908 exoplanetas descobertos dos 3989 exoplanetas confirmados 7 .

O primeiro exoplaneta detectado foi o HD $209458 \mathrm{~b}$ em novembro de 1999. A primeira detecção deste exoplaneta foi feita pela técnica de Velocidade Radial por [9] e no mesmo ano foi detectado pelo método de trânsito [10].

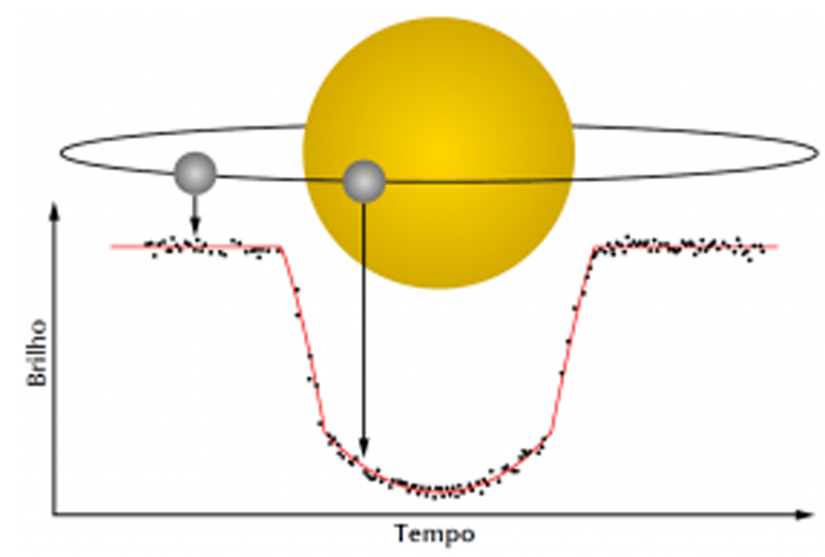

Figura 2: Representação artística de um planeta transitando uma estrela. Ao passar na frente da estrela na nossa linha de visada, o planeta bloqueia uma pequena fração $(<1 \%)$ da luminosidade da estrela causando uma queda nesse brilho que é visto no gráfico em forma de U. Adaptado de http://www2.ifa.hawaii.edu/ newsletters/article. $c f m ? a=407 \& n=34$.

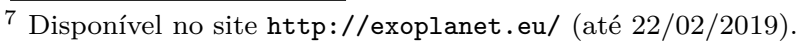


Ao transitar em frente à estrela hospedeira, o exoplaneta cruza o disco estelar na direção do observador (linha de visada) causando uma ligeira diminuição do brilho da estrela. Essa diminuição periódica do brilho nos permite inferir uma série de parâmetros físicos do sistema planetário, entre eles raio do planeta, parâmetros orbitais como período, semieixo e ângulo de inclinação.

\section{Detecção de manchas a partir de trânsitos planetários}

As manchas solares (Figura 3) são regiões na superfície do Sol que constituem regiões ativas. São áreas mais frias que o restante da superfície e acredita-se que sua origem está relacionada com a presença de intensos campos magnéticos [11].

As manchas solares são fenômenos conhecidos há bastante tempo por diversos povos. No oriente, os primeiros a relatar essas manchas no Sol foram os chineses em 165 a.C ([12]; [13]). O estudo de manchas solares sob ponto de vista científico começa na Europa com o surgimento do telescópio em 1611 [14] para poucas observações.

A frequência de observações das manchas solares começa no período entre 1700-1748 onde foram relatados somente médias anuais de presença de manchas na superfície do Sol, entre 1749-1817 médias mensais estão disponíveis e valores diários (com muitos intervalos sem dados) desde 1818 [15]. E como resultado uma das primeiras características observada continuamente desde então é o número de manchas solares e os grupos de manchas solares [14].

Uma consequência direta dessas observações diárias do Sol foi a descoberta do ciclo solar 8 em 1843 por Heinrich

\footnotetext{
8 O ciclo de atividade solar, que apresenta uma duração média de cerca de 11 anos, caracteriza o período durante o qual alguns índices
}

Schwabe (1789-1875). Schwabe descobriu o ciclo de 11 anos em 1843, e o astrônomo suíço Rudolf Wolf (18161893) confirmou a descoberta de Schwabe procurando referências anteriores ao número de pontos e estabeleceu sua periodicidade em 11 anos. Wolf fez um gigantesco esforço para compilar e reconstruir a atividade solar [16].

Assim como as manchas no Sol, outras estrelas apresentam esses fenômenos as quais estão relacionados com a atividade estelar. Evidências de manchas em outras estrelas foram inicialmente detectadas por [17]. Segundo [18] provavelmente todas as estrelas fria: 99 , ou com uma região convectiva ${ }^{10}$ semelhante à do Sol, poderão apresentar manchas em sua superfície. A presença das manchas nas estrelas podem ser detectadas ao analisar os trânsitos planetários nas curvas de luz da estrela.

A detecção das manchas na superfície das estrelas obtidas das observações do telescópio Kepler é vista nas curvas de luz como um aumento de brilho durante o trânsito planetário. Ao transitar a estrela, o planeta pode passar em frente de uma região de mancha na superfície da estrela e na curva de luz um pequeno pico no fluxo durante o trânsito é observado. Isso ocorre devido a região das manchas serem mais frias que a superfície da estrela.

solares - número de manchas solares apresentam uma variação aproximadamente cíclica e em fase entre máximo e mínimo.

9 Estrelas apresentam um amplo domínio de cores, que refletem a temperatura em suas atmosferas em concordância com lei de Wien. A cor de uma estrela é determinada seu espectro visível. Estrelas azuis são as mais quentes, as vermelhas as mais frias. No caso de estrelas, "fria"significa temperaturas da ordem de 2000 ou $3000 \mathrm{~K}$. 10 A Zona Convectiva é a responsável pelo transporte de energia gerada no núcleo até a superfície do Sol. Nessa região, que possui cerca de $15 \%$ do raio solar, o transporte de energia é dado pelas correntes de convecção que se formam nas porções de gás. Logo abaixo dessa região encontra-se a Zona Radiativa, onde a energia flui por radiação (emissão de fótons) sem que ocorra deslocamento de matéria. Crédito: https://lief.if.ufrgs.br/pub/cref/n20_Darroz/texto_sol.html

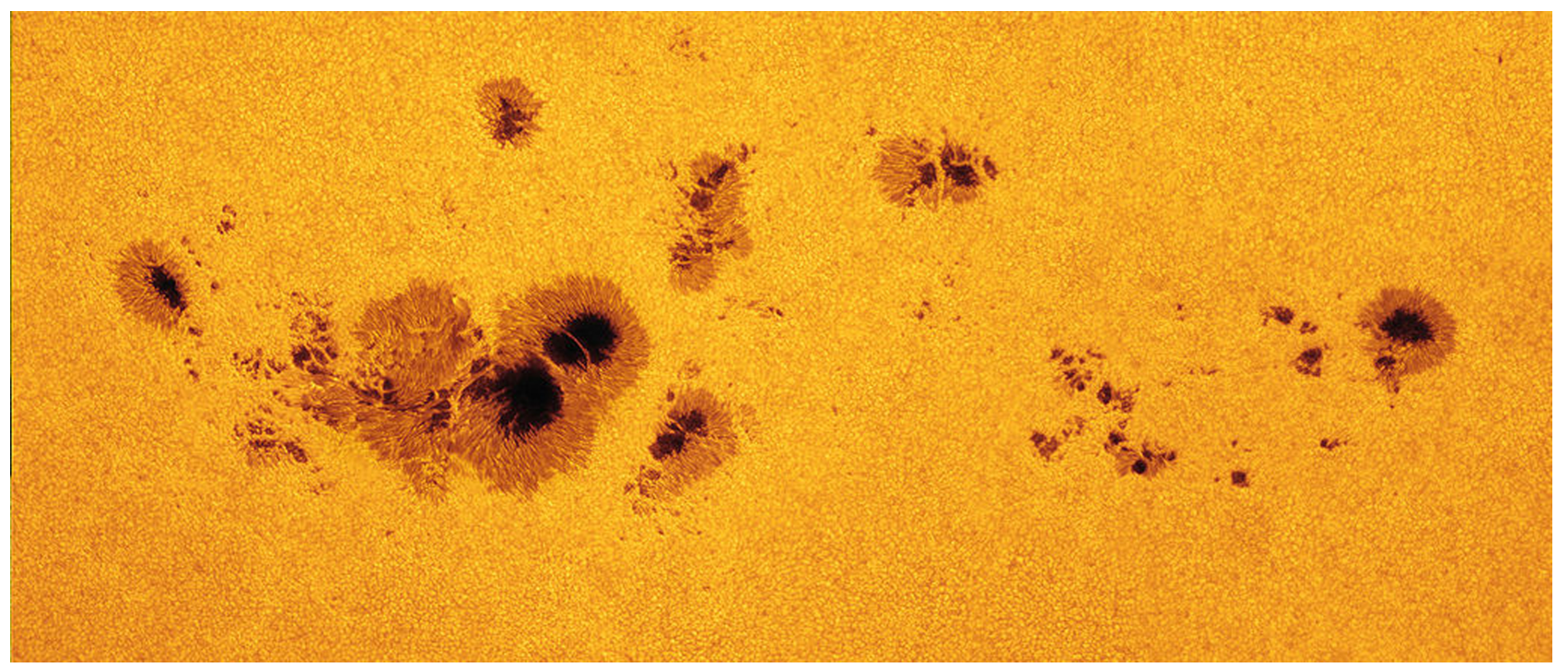

Figura 3: Um grupo de manchas solares em uma região ativa observado em 7 de julho de 2012. As regiões mais escuras são denominadas de Umbra e as regiões escuras com tons mais claros são denominadas de Penumbra. Fonte: NASA. 

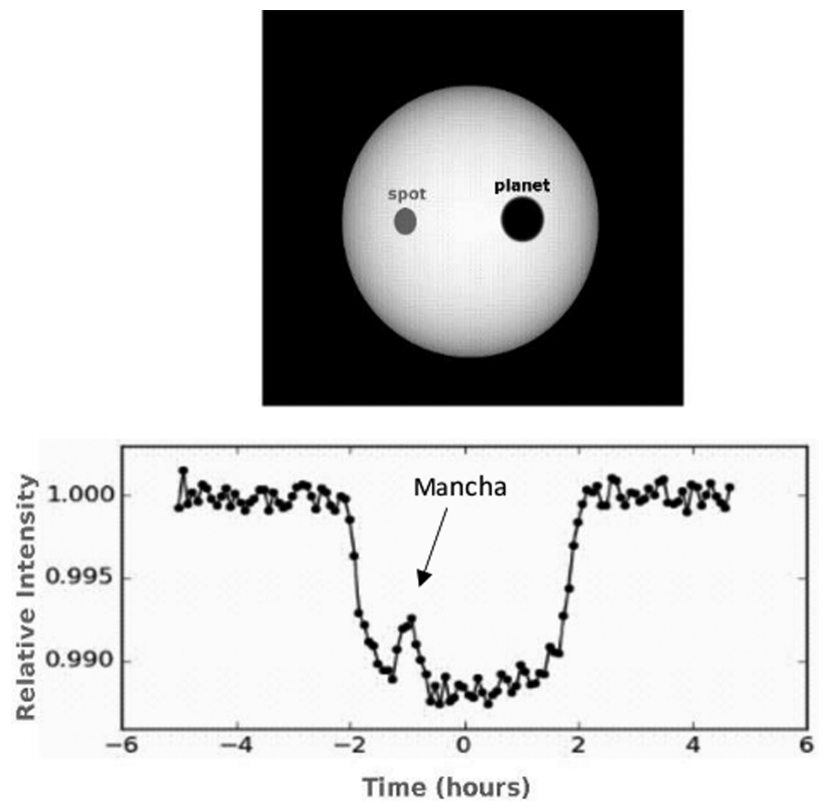

Figura 4: No painel superior temos a simulação de uma imagem sintetizada bidimensional de uma estrela com mancha, e o planeta cruzando-a durante o trânsito. No painel inferior, temos o trânsito do planeta com um pico referente a presença de mancha. Crédito: Adaptado de [19].

Um exemplo dessa observação pode ser visto no painel inferior da Figura 4.

\section{Modelo utilizado nas manchas estelares e sua aplicação}

O modelo apresentado neste artigo para o estudo de manchas estelares é o modelo de [19], que foi proposto pela $1^{\mathrm{a}}$ vez para utilizar um exoplaneta como ponta de prova para detectar a presença de manchas na superfície de estrelas. De forma sucinta, o modelo consiste em caracterizar fisicamente manchas em estrelas do tipo solar (F, G, K) observadas pelo satélite Kepler. Este método gera uma imagem bidimensional (ver Figura 4, painel superior) sintetizada da estrela levando-se em conta o obscurecimento de limbo, explicado a seguir.

\subsection{Obscurecimento de limbo}

A temperatura efetiva das estrelas é dada pelo brilho superficial do disco como um todo, porém a distribuição de temperatura sobre o disco fotosférico não é uniforme. Nas bordas das estrelas ocorre uma diminuição de brilho definida como obscurecimento de limbo. O fenômeno de obscurecimento de limbo se dá pelo efeito de variação de brilho decorrente da variação de temperatura que ocorre na fotosfera. A partir da base da fotosfera a temperatura diminui até a base da cromosfera. Esse gradiente de temperatura encontrado na fotosfera é devido a um efeito geométrico detalhado na Figura 5. O observador ao direcionar o campo de visão para parte central do disco, é observado as camadas mais profundas, mais quentes e brilhantes da fotosfera. Assim, as contribuições maiores nas observações vêm das camadas mais externas, mais frias e menos brilhantes.

O brilho da estrela em função da distância ao centro do disco é dada por:

$$
\frac{I(\mu)}{I(1)}=1-u_{1}(1-\mu)-u_{2}(1-\mu)^{2}
$$

Onde $\mu$ é o cosseno do ângulo entre a linha de visada e a normal à superfície local da estrela e $u_{1}$ e $u_{2}$ são os coeficientes de obscurecimento de limbo e I(1) é o brilho no centro do disco . Ao transitar a estrela, a posição do planeta é calculada para cada intervalo de tempo de acordo com os seguintes parâmetros orbitais: período, semieixo maior $a$ e o ângulo de inclinação $i$ que são inicialmente obtidos a partir dos dados disponíveis na literatura. Tais parâmetros são ajustados pelo mínimos quadrados $\chi^{2}[21]$.

\subsection{Extração e normalização dos trânsitos}

Neste trabalho apresentamos a aplicação do modelo de manchas para a estrela Kepler-289. A Kepler-289 é uma estrela do tipo solar $(\mathrm{G})$ com massa $1,08( \pm 0,02)$ solar ${ }^{11}$ e raio $1( \pm 002)$ raio solar com idade de $0,65( \pm 0,44)$ Ganos e uma temperatura efetiva de $5990 \mathrm{~K}( \pm 40)$, possui 3 planetas detectados: Kepler-289 b (tipo superterra), c (tipo netuniano) e d (tipo Júpiter quente) [22].

A primeira etapa para modelar os trânsitos planetários é fazer o download das curvas de luz com trânsitos no site do MAST ${ }^{12}$ ou utilizar o pacote de rotinas do $\mathrm{PyK}^{13} \mathrm{em}$ python com vários exemplos de rotinas para se trabalhar com os dados do Kepler. As curvas de luz são arquivos com muitos pontos, e para facilitar a análise do trânsito é realizado um corte na curva de luz extraindo o período de interesse, ou seja, o intervalo na curva de luz com a presença do trânsito. O resultado é apenas o período de tempo onde o trânsito é detectado.

Após o download da curva de luz com o trânsito do exoplaneta (Figura 6 painel superior), é necessário extrair o período de interesse. Para isso, basta realizar o corte nos dados, à direita e à esquerda do trânsito na curva de luz, restando apenas o trânsito. Mostrado na Figura 6 painel inferior.

A Figura 6 (painel inferior) mostra agora o resultado do recorte do trânsito do exoplaneta Kepler-289 d obtido da curva de luz da Kepler-289. Na Figura 6 os dados apresentam uma tendência de longo tempo fora do trânsito. É necessário remover essa tendência antes de ajustar o trânsito com os 5 parâmetros (semieixo maior, raio do planeta, ângulo de inclinação, e coeficientes de escurecimento de limbo). A variação lenta da curva de luz é

11 Massa do Sol $=1,988 \times 10^{30} \mathrm{~kg}$

12 Nesse site é disponibilizado um tutorial passo a passo para obter todas as informações no MAST. https://www.cfa.harvard.edu/ avanderb/tutorial/tutorial2.html

13 http://pyke.keplerscience.org/api/lightcurve.html 


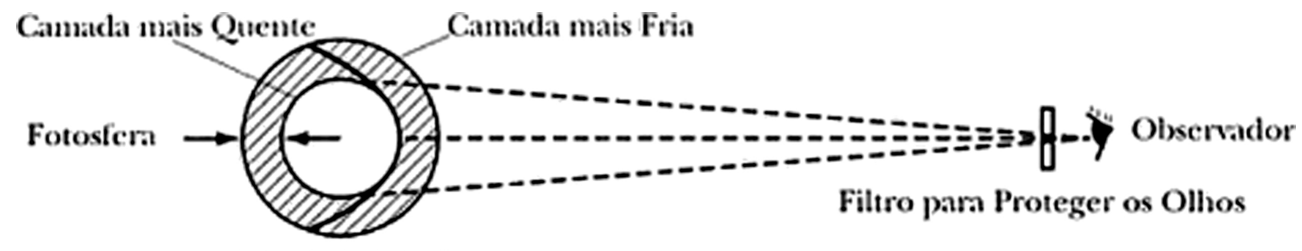

Figura 5: Esquema geométrico de observação do efeito da contribuição do obscurecimento de limbo. Adaptado de [20].
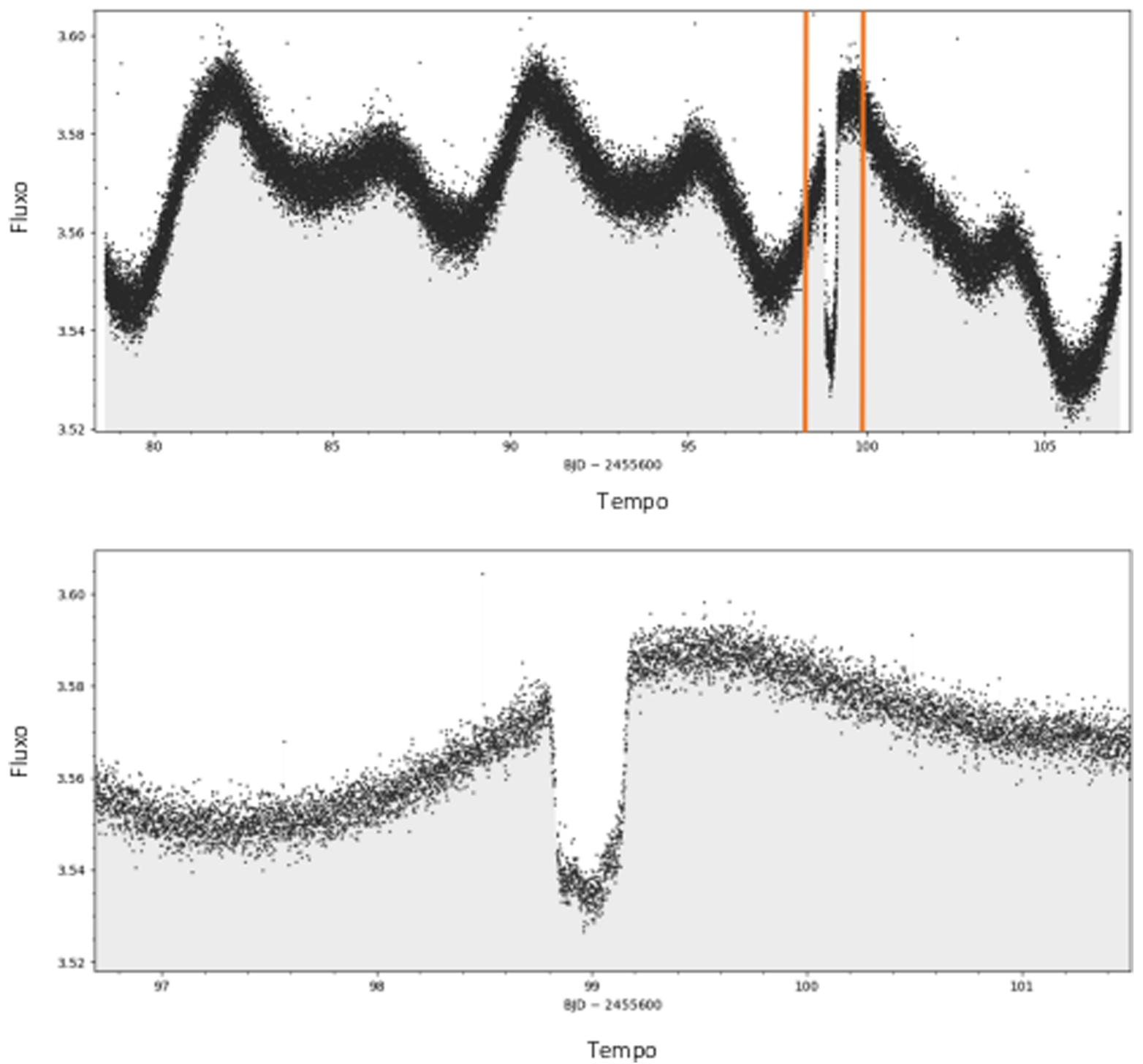

Figura 6: Painel superior: curva de luz obtida pelo telescópio Kepler com o trânsito do exoplaneta disponível para baixar no site do MAST. O intervalo entre as linhas é o trânsito na curva de luz. Painel inferior: recorte do trânsito.

eliminada ajustando e subtraindo um polinômio de $3^{\text {a }}$ ordem. Resultado mostrado na Figura 7. Em seguida é realizado o ajuste no trânsito mostrado na Figura 8 sem levar em consideração a presença de manchas.

\subsection{Modelagem dos trânsitos}

Nas análises das curvas de luz da Kepler-289 foram detectados apenas 3 trânsitos planetários do exoplaneta Kepler-289 d devido ao longo do período orbital de 125 dias. Os ajustes foram feitos para os parâmetros do planeta Kepler-289 d [22]. Os parâmetros para o ajuste estão descritos na Tabela 1 e na Figura 9 é possível ver os trânsitos detectados e os ajustes. Inicialmente, ajusta-se o modelo sem manchas e é retirada uma média de todos os trânsitos, então é subtraído da curva de luz o resíduo onde são ressaltados alguns picos. Os picos nos resíduos com intensidade maior que 3 sigmas são entendidos como manchas na superfície da estrela ocultadas pelo planeta durante seu período orbital do trânsito. 


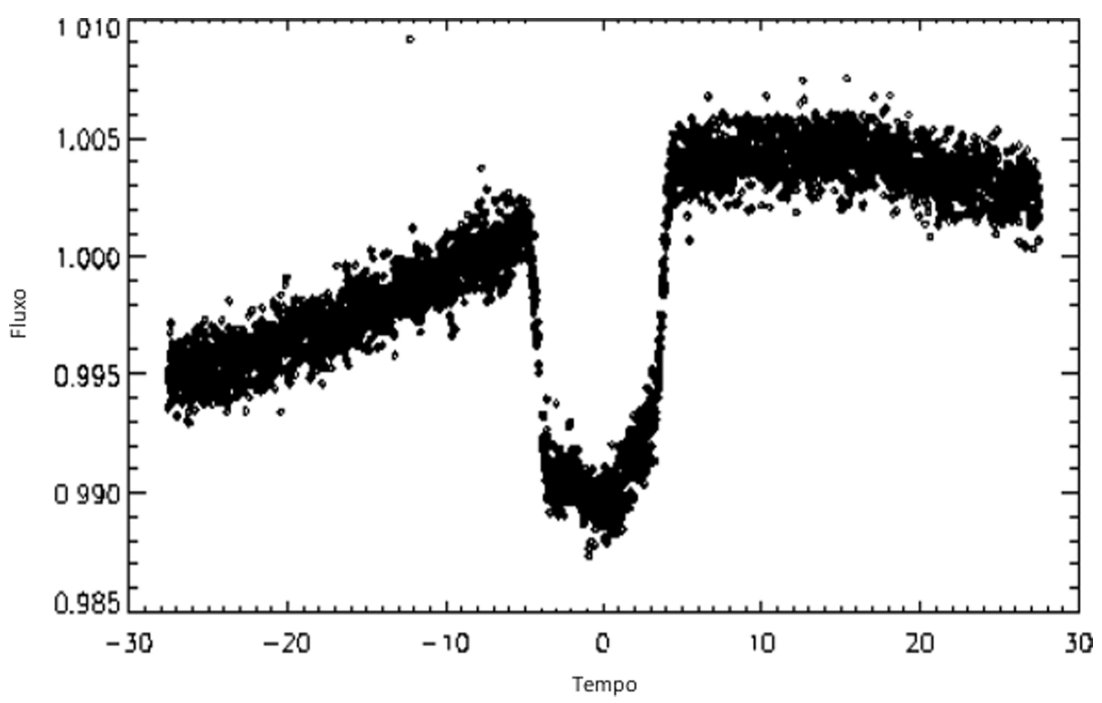

Figura 7: Trânsito obtido depois de realizar o corte na curva de luz.

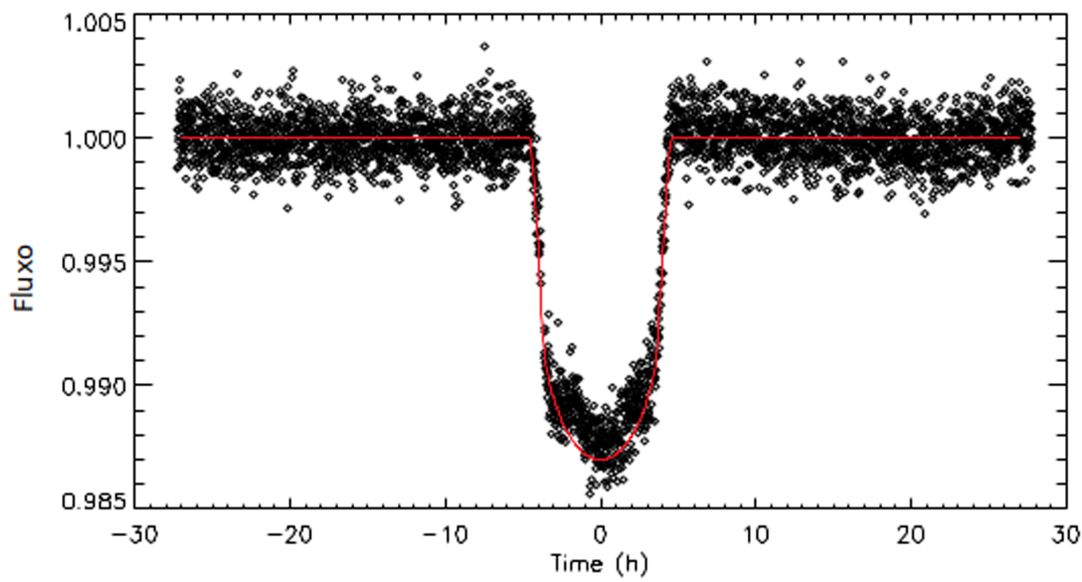

Figura 8: Resultado do modelo sem manchas. A linha vermelha é o ajuste realizado pelo método de trânsitos.

Tabela 1: Parâmetros do modelo de estrela sem manchas e os coeficientes de obscurecimento de limbo. O raio do planeta e semieixo orbital são dados em relação ao raio da estrela [22]

\begin{tabular}{lc}
\hline Obscurecimento de limbo $\mathrm{u}_{1}$ e $\mathrm{u}_{2}$ & 0.46 e 0.046 \\
\hline Raio do planeta & 0.102 \\
\hline Semieixo orbital & 112.0 \\
\hline Ângulo de inclinação & $89.79( \pm 0.0017)$ \\
\hline Período do Planeta & $125.85( \pm 0.0076)$ dias \\
\hline
\end{tabular}

Obtivemos os resíduos para os 3 trânsitos do Kepler289 d (Painel inferior da Figura 9), podem-se notar excessos de intensidade durante os trânsitos sendo interpretados como assinatura da presença de manchas na superfície da estrela detectadas pela passagem do exoplaneta na frente do disco estelar durante seu período orbital. O número total de picos foram 9 , três em cada trânsito, entendidos como a mesma quantidade de manchas.

\section{Parâmetros físicos das manchas}

Dos excessos detectados nos resíduos é possível estimar parâmetros físicos para as manchas. Esses resíduos foram modelados considerando os seguintes parâmetros: tamanho, intensidade e localização das manchas na superfície da estrela Kepler-289. A mancha estelar é modelada como um disco circular sendo possível obter:

- O tamanho da mancha em unidade do raio planetário $\mathrm{R}_{\mathrm{p}}$;

- Intensidade: intensidade da mancha em relação ao brilho do centro da estrela;

- Posição: longitude e latitude no disco estelar.

A longitude da mancha, é obtido do tempo aproximado do pico das manchas nos resíduos. Onde o $t$ é dado em 

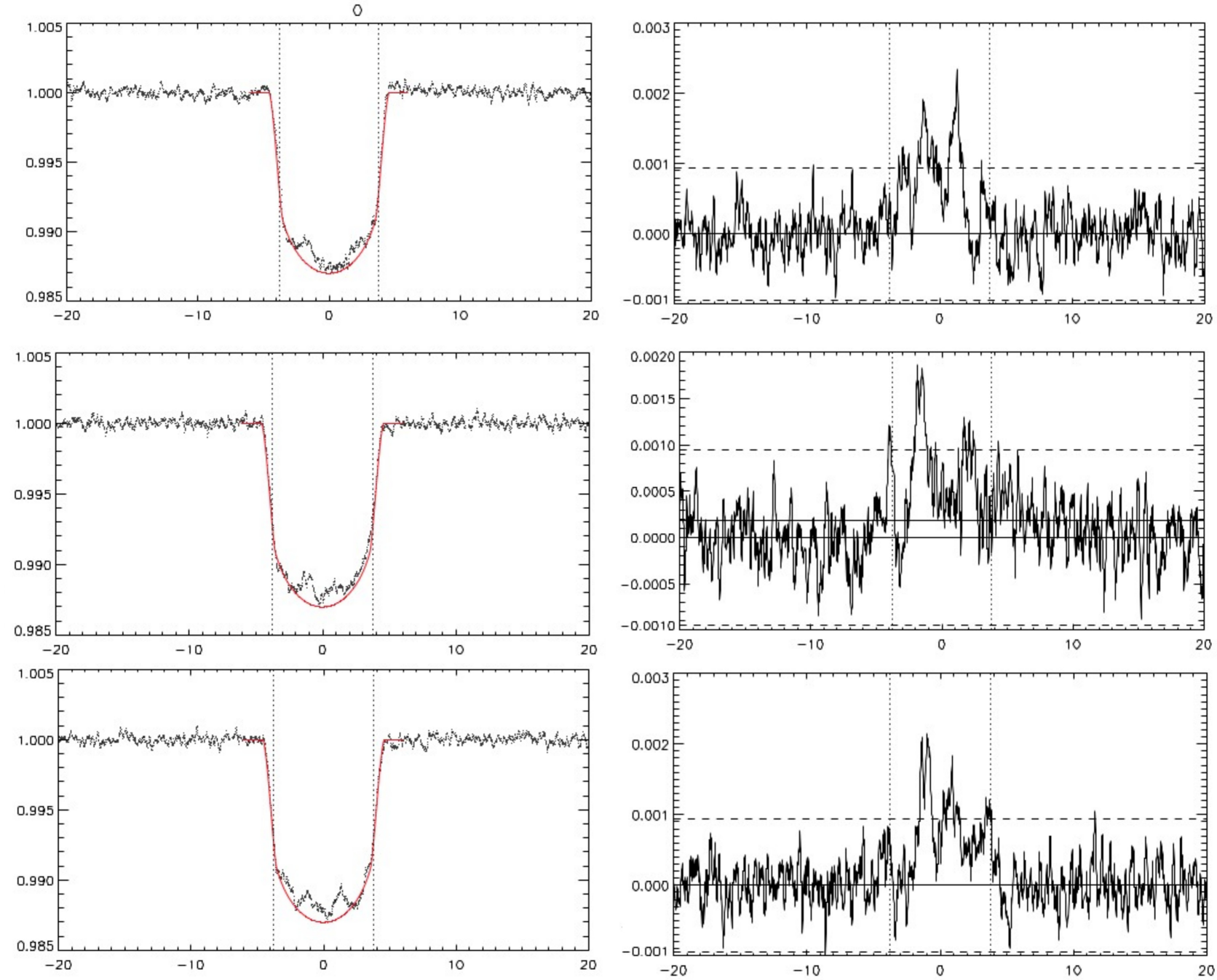

Figura 9: Curva de luz dos trânsitos de Kepler-289d (linha preta), com os ajustes do modelo sem manchas (linha vermelha. Em seguida é realizado uma subtração dos pontos no trânsito (linha vermelha) e o que sobra são os resíduos de pontos durante o trânsito, vistos nos painéis inferiores. $O$ excesso visto nos resíduos com picos maiores são entendidos como as manchas estelares. Onde os eixos x e y são respectivamente tempo em Barycentric Julian Date (BJD) e fluxo normalizado. O código para modelagem das manchas foi escrito em linguagem IDL (Interactive Data Language)

horas, de acordo com a seguinte relação:

$$
l g=\operatorname{asen}\left(\frac{\operatorname{acos}\left(90^{\circ}-360^{\circ}\right)\left(\frac{t}{24}\right)}{\cos (\text { lat })}\right)
$$

onde, lg é a longitude da mancha, a é o semieixo maior cos é cosseno, sen é o seno e $\mathrm{P}_{\text {orb }}$ é o período orbital do planeta e lat é a latitude da projeção do trânsito no disco estelar dada por:

$$
l a t=\operatorname{asen}\left(\frac{\mathrm{a}}{R * \operatorname{cosi}}\right)
$$

Sendo $R_{*}$ é o raio da estrela, e $i$ é o ângulo de inclinação. A temperatura da mancha é obtida levando-se em consideração que tanto a estrela como a mancha emitem radiação como um corpo negro. Toda a radiação emitida pelo corpo negro é devida à sua temperatura. A intensidade específica monocromática (energia por unidade de comprimento de onda, por segundo, por unidade de área e por unidade de ângulo sólido) de um corpo que tem uma temperatura uniforme $\mathrm{T}$ e está em equilíbrio termodinâmico com seu próprio campo de radiação, é dada pela Lei de Planck. Assim, a intensidade (I) de radiação é dada por:

$$
I=\frac{8 \pi h \nu^{3}}{c^{3}} \frac{1}{e^{\frac{h \nu}{K_{b} T_{e}}}-1}
$$

onde $K_{b}$ e h são as constantes de Boltzmann e Planck respectivamente, $\nu$ é a frequência e $T_{e}$ é a temperatura efetiva da fotosfera estelar. A fração de intensidade da

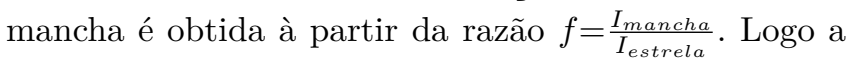
temperatura da mancha será obtida pela seguinte equa- 
ção:

$$
T=\frac{h \nu}{K_{b}}\left[\ln \left(1+\frac{e^{\frac{h \nu}{K_{b} T_{e}}}-1}{f}\right)\right]^{-1}
$$

A Figura 10 mostra os resultados dos ajustes dos resíduos obtidos da curva de luz da Kepler-289. Para uma melhor compreensão é mostrada a imagem bidimensional de uma estrela com 3 manchas na superfície, e ao lado o resultado dos ajustes com os três picos obtidos pelos resíduos. A média dos valores obtidos para raio, intensidade e temperatura, são listados na Tabela 2. Também são listados o intervalo de longitudes das manchas e a latitude projetada do trânsito.
Tabela 2: Valores médios dos parâmetros físicos obtidos das manchas para a Kepler-289. Onde $R_{p}$ é o raio do planeta e $I_{c}$ é luminosidade central da estrela.

\begin{tabular}{lc}
\hline Raio $\left(R_{p}\right)$ & $0.56 \pm 0.15\left(R_{p}\right)$ \\
\hline Intensidade & $0.40 \pm 0.20\left(l_{c}\right)$ \\
\hline Longitude & $-80^{\circ}$ a $60^{\circ}$ \\
\hline Latitude & $-20.5^{\circ}$ \\
\hline Temperatura & $4700 \pm 800(\mathrm{~K})$ \\
\hline
\end{tabular}
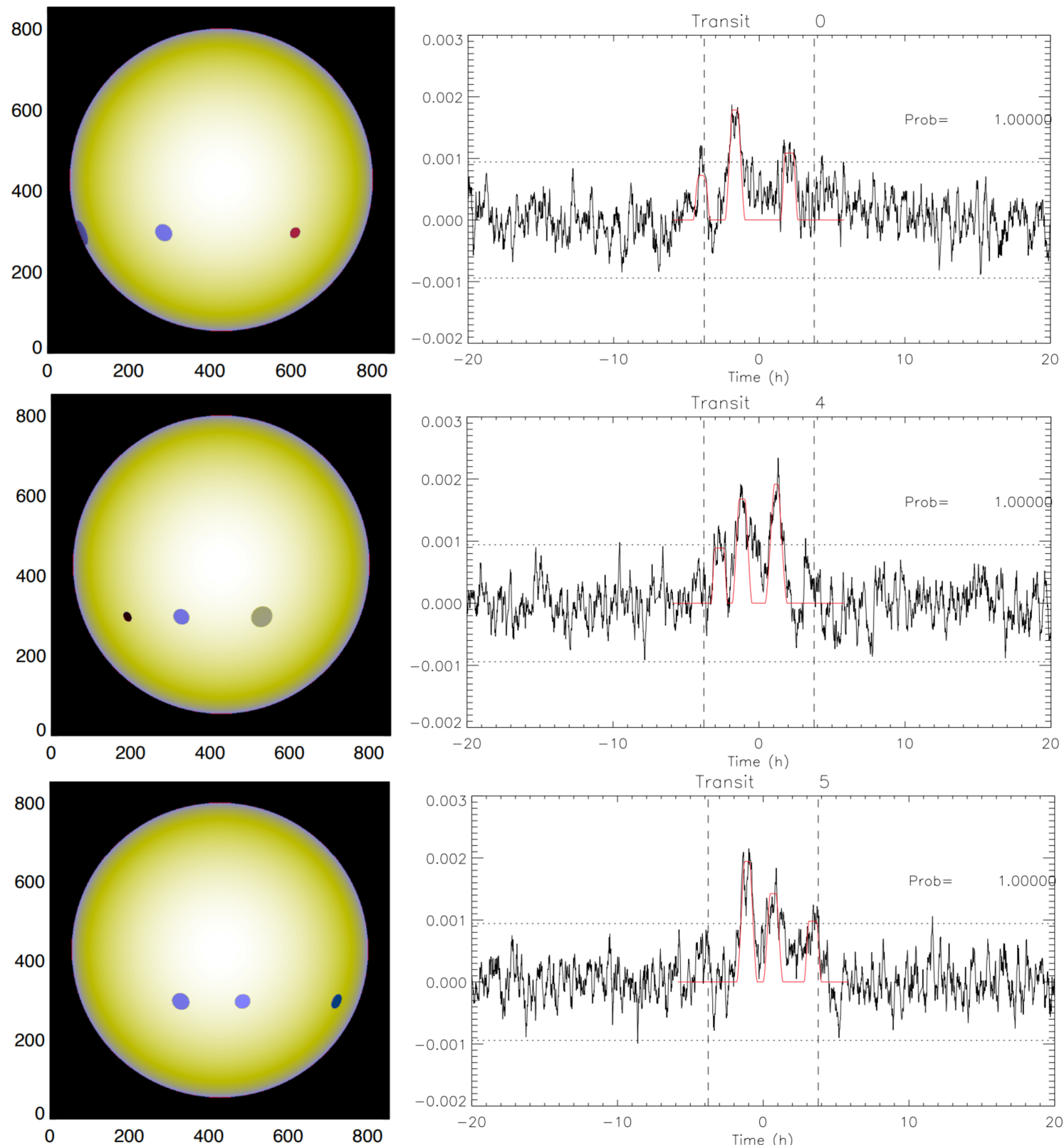

Figura 10: Imagem bidimensional de uma estrela com a presença de 3 manchas na sua superfície e ao lado o modelo do ajuste dos excessos apresentados na curva vermelha para os 3 trânsitos do Kepler-289 d. 


\section{Determinação do período de rotação da estrela kepler-289}

Séries temporais fotométricas de alta precisão obtidas com o telescópio Kepler permitiram a detecção de períodos de rotação para muitas estrelas ([4];[5];[23]) através de métodos baseados no periodograma Lomb-Scargle ([24]; [25]). Este algoritmo pode ser baseado tanto numa modificação da Transformada Discreta de Fourier como também na regressão de mínimos quadrados dos dados decompostos em funções sinusoidais com um intervalo de frequências.

Através do periodograma Lomb-Scargle é possível medir a rotação estelar das estrelas. A rotação estelar é um dos parâmetros de grande importância no estudo da evolução das estrelas. A partir dele é possível obter informações sobre idade, transferência de momentum angular, desaceleração de estrelas em relação ao tempo de vida, campos magnéticos e etc. ([21]; [26]).

O modelo retratado neste trabalho é de grande eficácia no estudo de rotação estelar, como também na obtenção da rotação diferencial em estrelas do tipo solar utilizando a posição das manchas na superfície detectadas pelos trânsitos planetários.

O resultado do cálculo do período de rotação médio da estrela Kepler-289 e visto no espectro de potência na Figura 11. Neste gráfico pudemos notar um pico em 8,76 dias, que então é adotado como o período de rotação estelar médio da estrela.

\section{Considerações finais}

Este trabalho teve como objetivo reportar o estudo de manchas em estrelas utilizando o método de trânsito planetário. Como exemplificação deste método utilizamos a estrela Kepler-289. Analisando os trânsitos detectados em suas curvas de luz foi possível obter as características físicas das manchas em sua superfície como intensidade, temperatura e tamanho. Além dessas características obtivemos o período de rotação estelar médio da estrela Kepler-289 através do periodograma de Lomb-Scargle.

A abordagem deste trabalho pode contribuir para uma interdisciplinaridade que podem ser utilizadas em disciplinas introdutórias no ensino de programação em cursos de Física, Ciências Naturais, Astronomia e Ciência da Computação, principalmente em atividades que exijam dos alunos começar a criar pequenos programas em alguma linguagem de programação (Neste trabalho foi utilizado Python), realizar buscas em bancos de dados ou iniciar atividades que envolvam análises de séries temporais. Os dados do telescópio Kepler são de fácil acesso e podem ser analisados a partir de diversas ferramentas públicas como: Pyke; lightkurve, K2mosaic e etc. Todos disponíveis no keplerscience ${ }^{14}$ (Ver no Apêndice A e C).

Para atividades iniciais sem programação é possível explorar a página Nasa exoplanet archive ${ }^{15}$ sendo possível obter diversas informações sobre exoplanetas, fazer ajustes de curva de luz, calcular rotação estelar média e realizar download dos dados da missão Kepler (ver no

\footnotetext{
14 https://keplerscience.arc.nasa.gov/software.html 15 https://exoplanetarchive.ipac.caltech.edu/
}

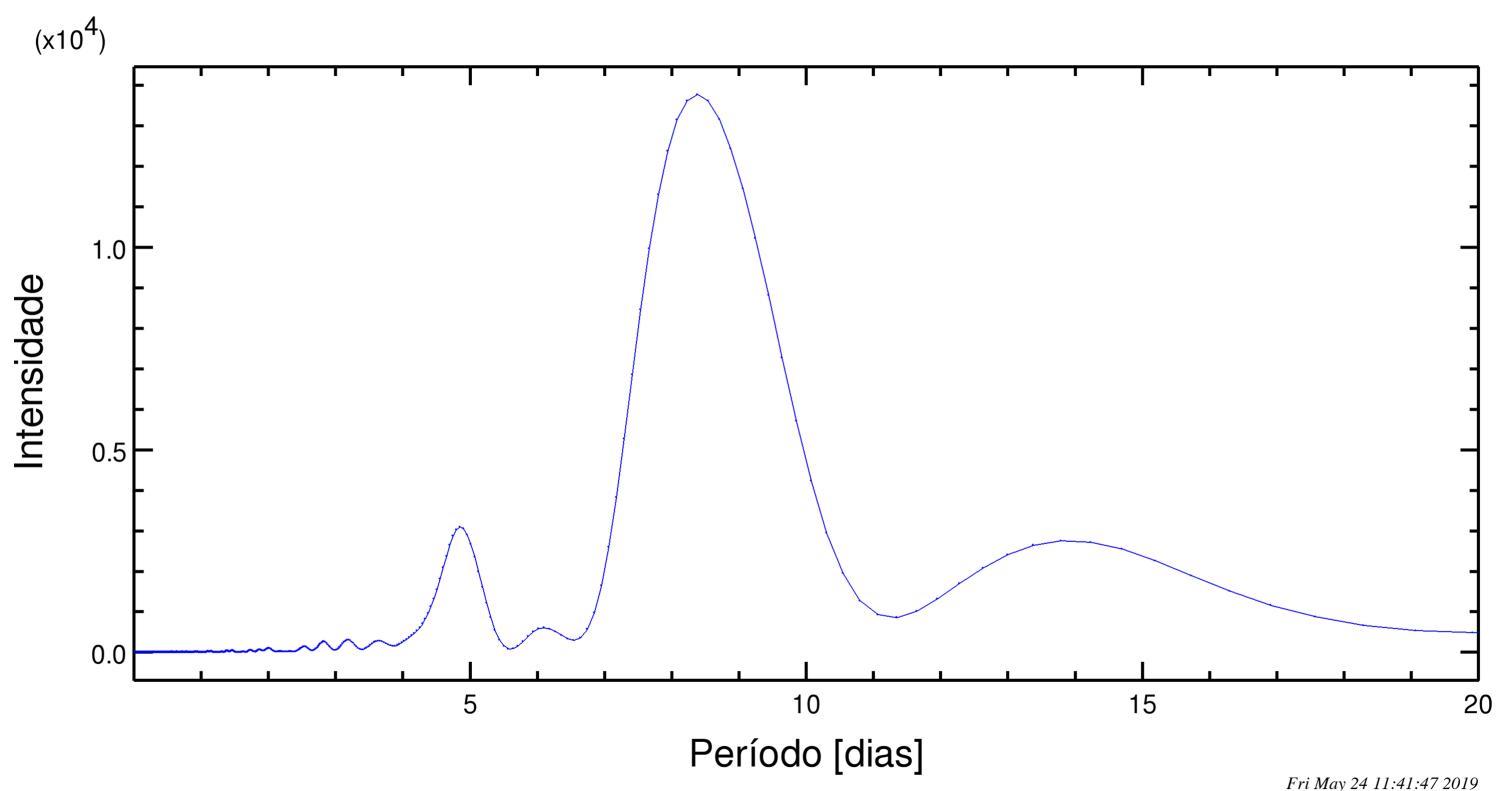

Figura 11: Periodograma de Lomb-Scargle com pico em 8,76 dias, considerado o período de rotação médio da estrela Kepler-289. 
Apêndice B). Esperamos que o trabalho possa despertar o interesse pela astronomia tanto de alunos como de professores da educação básica e do nível superior a fim de utilizar estudos de astronomia de maneira interdisciplinar em sala de aula, proporcionando aos alunos novos conhecimentos científicos das práticas e métodos existentes de produção científica com dados públicos de grandes missões espaciais.

\section{Material Suplementar}

O seguinte material suplementar está disponível online: Apêndice $A$

Apêndice B

Apêndice $\mathrm{C}$

\section{Referências}

[1] J.B. William, Rep. Prog. Phys. 79, 036901 (2016).

[2] J.M. Jenkins, J.D. Twicken, N.M. Batalha, D.A. Caldwell, W.D. Cochran, M. Endl, D.W. Latham, G.A. Esquerdo, S. Seader, A. Bieryla et al., AJ 150, 56 (2015).

[3] W.J. Chaplin, T. Appourchaux, Y. Elsworth, R.A. García, G. Houdek, C. Karoff, T.S. Metcalfe, J. MolendaŻakowicz, M.J.P.F.G. Monteiro, M.J. Thompson et al., The Astrophysical journal letters 713, L169 (2010).

[4] T. Reinhold, A. Reiners e G. Basri, Astronomy \& Astrophysics 560, A4 (2013)

[5] A. McQuillan, T. Mazeh e S. Aigrain, The Astrophysical Journal Supplement Series 211, 24 (2014).

[6] H. Maehara, T. Shibayama, S. Notsu, Y. Notsu, T. Nagao, S. Kusaba, S. Honda, D. Nogami e K. Shibata, Nature 485, 478 (2012).

[7] M.R. Haas, N.M. Batalha, S.T. Bryson, D.A. Caldwell, J.L. Dotson1, J. Hall, J.M. Jenkins, T.C. Klaus, D.G. Koch e J. Kolodziejczak, The Astrophysical Journal Letters 713, L115 (2010).

[8] R. Estrela e A. Valio, The Astrophysical Journal 831, 57 (2016).

[9] G.W. Henry, G.W. Marcy, R.P. Butler e S.S. Vogt, The Astrophysical Journal Letters 529, L41 (2000).

[10] D. Charbonneau, T.M. Brown, D.W. Latham, e M. Mayor, The Astrophysical Journal Letters 529, L45 (2000).

[11] H. George, The astrophysical journal 28, 315 (1908).

[12] A.D. Wittimann e Z.T. Xu, Astronomy and Astrophysics Supplement Series 70, 83 (1987).

[13] H. David, Living Reviews in Solar Physics 1, 315 (2010).

[14] E. John, Science 192, 1189 (1976).

[15] J. Vaquero, Advances in Space Research 40, 929 (2007).

[16] D. V. Hoyt e K. H. Schalten, The role of the sun in climate change (Oxford University Press, New York, 1997), v. 1, p. 37.

[17] K. Gerald, Publications of the Astronomical Society of the Pacific 59, 261 (1947).

[18] K.G. Strassmeier, E. Serkowitsch e T. Granzer, Astronomy and Astrophysics Supplement Series 125, 11 (1999).
[19] A. V. R. Silva, The Astrophysical Journal Letters 585, L147 (2003).

[20] A.C. Friaça, E. Dal Pino, L. Sodré e V.J. Pereira, Astronomia: uma visão geral do universo (EDUSP, São Paulo, 2000) v. 1, p. 88.

[21] A. Valio, R. Estrela, Y. Netto, J.P. Bravo e J.R. Medeiros, The Astrophysical Journal 835, 294 (2017).

[22] J.R. Schmitt, E. Agol, K.M. Deck, L.A. Rogers, J.Z. Gazak, D.A. Fischer, J. Wang, M.J. Holman, K.J. Jek, C. Margossian et al., The Astrophysical Journal 795, 167 (2014).

[23] R.A. García, T. Ceillier, D. Salabert, S. Mathur, J.L. Van Saders, M. Pinsonneault, J. Ballot, P.G. Beck, S. Bloemen, T.L. Campante et al., Astronomy \& Astrophysics 572, A34 (2014).

[24] N. Lomb, Astrophysics and space science 39, 447 (1976).

[25] J. Scargle, The Astrophysical Journal 263, 835 (1982).

[26] E. Courtney, The Astrophysical Journal 780, 159 (2014). 